\title{
Reconstrucción de la precipitación invierno-primavera con base en anillos de crecimiento de árboles para la región de San Dimas, Durango, México
}

\author{
Winter-spring precipitation reconstruction from tree-rings for San Dimas region, Durango, Mexico
}

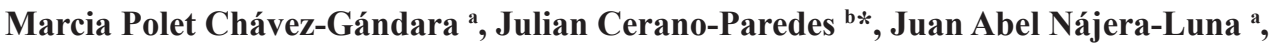 \\ Viridiana Pereda-Breceda a ${ }^{\text {a }}$ Gerardo Esquivel-Arriaga ${ }^{\text {b }}$, Rosalinda Cervantes-Martínez ${ }^{\text {b, }}$ \\ Víctor Hugo Cambrón-Sandoval c , Francisco Cruz-Cobos a, Sacramento Corral-Rivas a \\ a Instituto Tecnológico de El Salto, Mesa del Tecnológico s/n, El Salto, Pueblo Nuevo, Durango, México. \\ *Autor de correspondencia: ${ }^{b}$ INIFAP CENID-RASPA, km. 6,5 Margen Derecha del Canal Sacramento, \\ Gómez Palacio, Durango. México, C.P. 35140, cerano.julian@gmail.com \\ c Universidad Autónoma de Querétaro, Facultad de Ciencias Naturales, Querétaro, México.
}

\begin{abstract}
SUMMARY
The studies on reconstruction of paleoclimatic series play an important role in analyzing regional climate variability when instrumental information is not available. Tree-ring samples from stands of Pinus durangensis, Pinus cooperi and Pinus lumholtzii were used to generate chronologies in order to reconstruct and analyze the historical hydroclimatic variability for the region of San Dimas, Durango, Mexico. A principal component analysis (PCA) indicates that those series showed a common variability; subsequently, a regional series was developed covering a period of 294 years. An analysis of response function suggests that the most important climate variable influencing tree growth was the seasonal winter-spring precipitation (January-June), which accounted for $52 \%$ of the variability. Reconstruction of the variability of the winter-spring precipitation for the last three centuries was possible. Such a profile allowed us to identify important drought periods (1740-1746, 1766-1780, 1785-1786, 1819-1824, 1890-1900, 1935-1940, 1950-1957 and 2011-2015). Because of their extension, the periods 1766-1780, 1890-1900 and 1950-1957 were the longest. Based on historical records, four events of severe drought (1785-1786, 1935-1940, 1891-1902 and 1950) were associated with strong havoc in central Mexico and their effects were extended to northern Mexico. In addition, a wavelet coherence analysis suggests a strong relationship between the reconstructed precipitation (Winter-Spring) and NIÑO 3 SST (December to February) in phase from 1770 to 1978 with frequencies from 1 to 5 years, ENSO has significantly modulated climate variability in this region of the state of Durango.
\end{abstract}

Key words: chronologies, reconstructed precipitation, drought, ENSO.

\section{RESUMEN}

Los estudios de reconstrucción de series paleoclimáticas juegan un papel importante para analizar la variabilidad del clima regional cuando no se dispone de información instrumental. Muestras de anillos de árboles de rodales de Pinus durangensis, Pinus cooperi y Pinus lumholtzii fueron empleados para generar cronologías con el objetivo de reconstruir y analizar la variabilidad hidroclimática histórica para la región de San Dimas, Durango, México. Un análisis de componentes principales (PCA) determinó que las series presentan variabilidad común, se desarrolló una serie regional de 294 años. Un análisis de función de respuesta mostró que la variable climática que más ha influido en el crecimiento es la precipitación estacional invierno-primavera (enero-junio), que explicó el 52 \% de la variabilidad. Se logró reconstruir la lluvia invierno-primavera para los últimos tres siglos. Esta reconstrucción permitió identificar importantes periodos de sequía (1740-1746, 1766-1780, 1785-1786, 1819-1824, 1890-1900, 1935-1940, 1950-1957 y 2011-2015), por su extensión destacaron los periodos 1766-1780, 1890-1900 y 1950-1957. Con base en archivos históricos, se corroboraron cuatro eventos de sequía severa (1785-1786, 1935-1940, 1891-1902 y la década de 1950) que propiciaron fuertes estragos a la sociedad del centro del país y cuyos efectos se extendieron hasta el norte de México. Un análisis de coherencia de ondeletas mostró una fuerte relación entre la precipitación reconstruida (invierno-primavera) y NIÑO 3 SST (diciembre-febrero) en fase de 1770 a 1978 con frecuencias de 1 a 5 años, ENSO ha modulado significativamente la variabilidad climática en esta región del estado de Durango.

Palabras clave: cronologías, precipitación reconstruida, sequía, El Niño Oscilación del Sur.

\section{INTRODUCCIÓN}

Existe un interés creciente por el estudio de los cambios del clima global a través del tiempo y su posible com- portamiento futuro (Cerano et al. 2011). La información climática que cubre períodos de siglos o milenios soporta decisiones técnicas que se fundamentan en un conocimiento más amplio (Woodhouse y Lukas 2006). La forma más 
común de analizar la variabilidad del clima es a través del uso de información instrumental; sin embargo, el principal problema radica en su corta extensión y calidad. Para reconstruir series climáticas extensas se emplean diferentes fuentes alternativas naturales indirectas que registran dicha variación en el tiempo, tales como: anillos de crecimiento de los árboles, capas de hielo, polen fósil, sedimentos marinos y corales (Bradley 1999).

La dendrocronología emplea anillos de crecimiento de los árboles para reconstruir y analizar eventos climáticos pasados que alcanzan varios siglos, para algunos casos llegan a rebasar los 1000 años (Stahle et al. 2011). Es la fuente indirecta más apropiada de resolución anual para entender el comportamiento histórico del clima (Fritts 2001).

El análisis histórico de la variabilidad climática es fundamental para conocer su impacto en aspectos económicosociales, disponibilidad de agua, determinar las fluctuaciones climáticas de alta y baja frecuencia, causas de su variabilidad, así como analizar el presente e inferir posibles tendencias climáticas futuras (Villanueva et al. 2007). Reconstrucciones paleoclimáticas para el norte de México, han permitido analizar la recurrencia de eventos extremos de sequía (Cerano et al. 2011) con un efecto directo en la recarga de los mantos acuíferos y un impacto negativo sobre la sociedad (Cerano et al. 2012a), propiciando el abandono del campo y la migración hacia las grandes ciudades o hacia los Estados Unidos.

En México, en los últimos 15 años, se ha desarrollado una red de reconstrucciones paleoclimáticas de más de 120 series a lo largo del país, constituyendo una base de datos de alta resolución y gran importancia para reconstruir información histórica del clima en los últimos siglos, información que se puede incorporar a modelos de predicción bajo diversos escenarios climáticos (Cerano et al. 2009).

Sin embargo, a pesar de este arduo trabajo, las series se han desarrollado con base en los crecimientos anules de pocas especies, sobresaliendo Psedotsuga menziesii (Mirb.) Franco y Taxodium mucronatum Ten., México posee una gran diversidad de coníferas, 47 especies de pino (Perry et al. 1998), lo que indica, que existe mucho por explorar, analizar y determinar el potencial de nuevas especies para reconstruir el clima. Conocer el potencial dendroclimático de nuevas especies, representa una alternativa para reconstruir información climática por varios siglos en zonas del país donde se carece de datos instrumentales, datos esenciales para analizar el impacto, extensión y recurrencia de eventos extremos como sequías, y a su vez, determinar la influencia de fenómenos climáticos de circulación general en dicha variabilidad.

Por lo anterior, el presente estudio se plantea los siguientes objetivos: 1) desarrollar cronologías con base en los crecimientos anuales de Pinus durangensis Martínez, Pinus cooperi Blanco y Pinus lumholtzii Rob. et Fern., 2) determinar el potencial de las cronologías para reconstruir variables climáticas, 3) reconstruir la variabilidad climá- tica para esta región del norte de México y 4) analizar la influencia de El Niño Oscilación del Sur (ENSO, por sus siglas en inglés) en la variabilidad histórica del clima. Así mismo, se plantean las siguientes hipótesis de investigación: 1) los crecimientos anuales de Pinus durangensis, Pinus cooperi y Pinus lumholtzii presentan potencial para reconstruir la variabilidad climática que ha caracterizado en los últimos siglos a la región de San Dimas, Durango y 2) el fenómeno climático ENSO ha modulado la variabilidad climática histórica en esta región del norte del país.

\section{MÉTODOS}

Área de estudio. El estudio se desarrolló en la región del Río de Miravalles, municipio de San Dimas, al noroeste del estado de Durango, delimitada geográficamente por los paralelos $24^{\circ} 43^{\prime} 52^{\prime \prime}$ y $24^{\circ} 44^{\prime} 27^{\prime \prime} \mathrm{N}$ y $106^{\circ} 43^{\prime} 41^{\prime \prime}$ y $105^{\circ}$ 16 ' 48" O (figura 1). El área de estudio comprendió una extensión aproximada de 586.335 ha. Presenta un clima templado subhúmedo $(\mathrm{Cw})$ con temperaturas que oscilan entre $-16,5^{\circ} \mathrm{C}$ como mínima en el mes de diciembre, hasta los $33{ }^{\circ} \mathrm{C}$ en los meses de abril y mayo, una precipitación media anual que varía de 500 a $1.500 \mathrm{~mm}$, el gradiente altitudinal inicia en los 400 hasta los 3.262 m s.n.m, la altitud predominante oscila entre los 2.000 y 2.800 m s.n.m, el tipo de vegetación que predomina son los bosques de clima templado frío (INEGI 2010).

Métodos de campo. Con base en recorridos de campo se seleccionaron tres áreas de estudio, cada una dominada por una especie diferente, Pinus durangensis, Pinus cooperi y Pinus lumholtzii. Por medio de un muestreo selectivo se identificaron los individuos con las características adecuadas para desarrollar estudios dendroclimáticos tales como: árboles lo más longevos posible caracterizados por un fuste o ramas en forma de espiral, corteza delgada, copa reducida con ramas gruesas colgantes y con el menor disturbio posible. Se colectaron un total de 35 árboles por especie, se extrajeron de dos a tres muestras por árbol para lo cual se emplearon taladros de Pressler (figura 1A) de diferentes longitudes $(45,55$ y $65 \mathrm{~cm})$ y diámetros $(5$ y $12 \mathrm{~mm})$. Así mismo, en la medida de lo posible, se obtuvieron secciones transversales de árboles muertos con el apoyo de motosierra, esto con el objetivo de extender en el tiempo las series paleoclimáticas.

Método de laboratorio. En el Laboratorio de Dendrocronología del CENID-RASPA, INIFAP localizado en la ciudad de Gómez Palacio, Durango, las muestras se montaron y pulieron con diferentes granos de lija (de 120 a la 1.200) para lograr una mejor visibilidad de las estructuras de crecimiento anual. Con el apoyo de un estereomicroscopio con resolución de 10 a $30 \mathrm{X}$, se contó cada uno de los anillos de crecimiento y con base en técnicas dendrocronológicas estándar se pre fechó cada uno de los crecimientos (Stokes y Smiley 1996). 
Para cada anillo de crecimiento se midió la madera temprana, tardía y anillo total empleando un sistema de medición VELMEX de fase deslizable y precisión de $0,001 \mathrm{~mm}$. La calidad del fechado y la medición se verificó con el programa COFECHA (Holmes 1983).

Las tendencias biológicas (competencia, supresión, liberación) y las geométricas (incremento del área de fuste con la edad y disminución del crecimiento anual al distribuirse en una mayor superficie) para cada una de las muestras de las tres diferentes especies no relacionadas con clima, se removieron con el programa ARSTAN, para lo cual se insertó una curva exponencial negativa o línea recta a la serie de medición y posteriormente se dividió cada valor anual de medición entre el valor obtenido de la curva. Esto
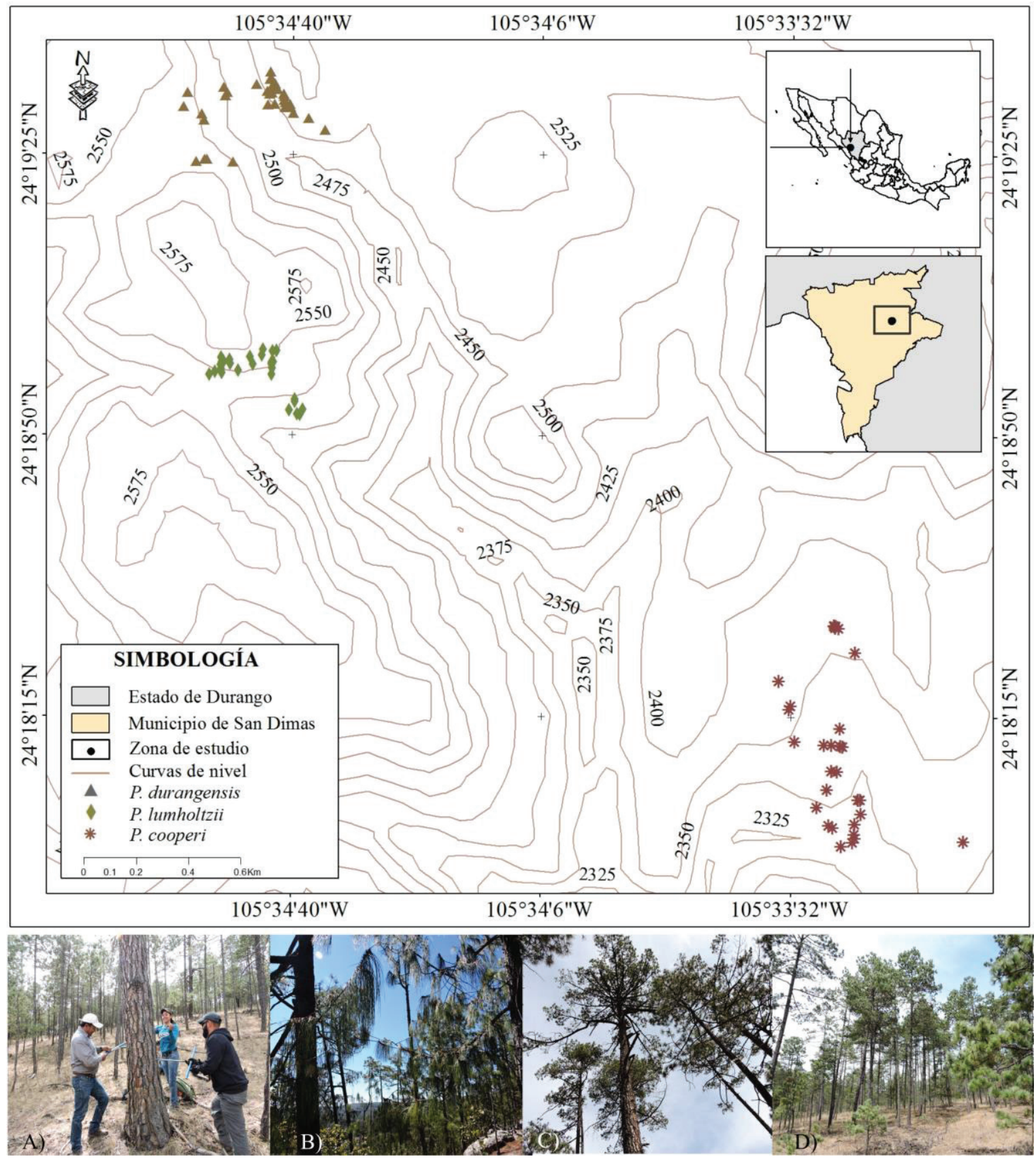

Figura 1. Distribución de las áreas de estudio en la zona del Río Miravalles, San Dimas, Durango. En la parte inferior se muestran cuatro fotografías, A) toma de muestras empleando taladros de Pressler, B) bosque de Pinus lumholtzii C) bosque de Pinus cooperi y D) bosque de Pinus durangensis.

Distribution of the sample points at Río Miravalles, San Dimas, Durango. At the bottom four photographs that show: A) taking the samples using an increment borer in stands of the three pine species, B) Pinus lumholzii C) Pinus cooperi and D) Pinus durangensis. 
creó tres series de índices normalizados (cronologías) con media de 1,0 y varianza homogénea (Fritts 2001).

Análisis de datos. Después de obtener las tres cronologías, se corrió un análisis de correlación y un análisis de componentes principales (PCA, por sus siglas en inglés) para el período común de las tres series (120 años; 1896-2015) para esto se empleó el programa STATISTICA Kernel Release 5.5 (Stat Soft Inc. 2000). Se determinó una variabilidad común entre cronologías y se desarrolló una serie paleoclimática regional.

Para conocer la variable climática y el período anual que más influye en el crecimiento de Pinus durangensis, Pinus cooperi y Pinus lumholtzii de la región de San Dimas, se utilizaron registros climáticos (precipitación, temperatura máxima y mínima) de las estaciones de El Salto y El Río de Miravalles, estaciones pertenecientes a la Comisión Nacional del Agua (CONAGUA) y las más cercanas a las áreas de estudio. Dado que las variables climáticas de ambas estaciones mostraron una correlación significativa $(P<0,05)$ con los índices de anillo de la cronología regional, las estaciones se combinaron para trabajar con datos promedio regionales, registros de 1991-2014 (24 años). Para determinar la variable climática que más influye en el crecimiento de las especies de pino, se corrió un análisis de función de respuesta entre los índices de anillo total, madera temprana y tardía y los datos climáticos (precipitación y temperaturas).

Una vez analizada la respuesta climática y definida la variable que más influye en el crecimiento, se procedió a desarrollar una ecuación de transferencia entre los datos observados y el índice de anillo, para ello se usó el programa STATISTICA Kernel Release 5.5 (Stat Soft Inc. 2000). Con este modelo se reconstruyó la precipitación para el período común que cubren dichos registros, y con la subrutina VERYFY5 del paquete de Programas Dendrocronológicos de la Universidad de Arizona (DPL por sus siglas en inglés) se aplicó una prueba de calibración a la mitad de los datos climáticos, tanto observados como reconstruidos para examinar la relación entre ambas variables; en una etapa posterior, a la mitad restante se le asignó una prueba de verificación (Fritts 2001). Por último, la ecuación se validó para el periodo total de datos de lluvia disponibles y se utilizó para generar la reconstrucción de la precipitación en la longitud total de la serie dendrocronológica regional, 294 años.
A la reconstrucción de precipitación de resolución anual o serie de alta frecuencia se le ajustó una curva decenal flexible o de baja frecuencia, para resaltar los eventos secos y lluviosos presentes en la reconstrucción. Las sequías detectadas en la reconstrucción se validaron con documentos históricos y estudios dendrocronológicos para el norte de México.

Finalmente, para analizar el impacto de ENSO sobre la variabilidad climática de esta región del estado de Durango, se relacionaron los índices de ancho de anillo y los índices NIÑO 3.4 SST (estimativos de la variabilidad del ENSO) mediante el programa MATLAB 6.5 (MathWorks 2002). Para tal efecto, se utilizaron gráficos de resolución anual y sus espectros potenciales de ondeleta.

\section{RESULTADOS}

Se logró fechar un total de 130 núcleos de crecimiento de tres diferentes especies: 47 de Pinus durangensis, 44 de Pinus cooperi y 41 de Pinus lumholtzii. La intercorrelación entre series fue estadísticamente significativa para cada una de las especies (cuadro 1), superando el parámetro establecido por el programa COFECHA (Holmes 1983), que establece como mínima correlación significativa entre muestras $r=0,3281(P<0,01)$. Ello indica que las tres especies poseen una buena sensibilidad a los cambios ambientales (cuadro 1) y presentan potencial para fines de reconstrucción de series paleoclimáticas.

Se generaron tres cronologías de diferente longitud (figura 2, cuadro 1), 120 años para Pinus durangensis que cubrió el período de 1896-2015; 126 años para Pinus cooperi (1890-2015) y 294 años para Pinus lumnholtzii (1722 al 2015).

Se determinó una correlación significativa $(P<0,01)$ entre las series dendrocronológicas generadas y una distribución normal de los datos (figura 3A). Así mismo, el PCA indicó que las tres cronologías se agruparon en el PC1 (figura 3B). Se determinó una variabilidad común y se logró desarrollar una cronología regional para los pasados tres siglos que mostró la variabilidad climática de baja y alta frecuencia para la región de San Dimas, Durango.

Función de respuesta. Este análisis indicó que los meses individuales de precipitación de enero a julio, presentaron una relación positiva con la cronología regional de anillo

Cuadro 1. Parámetros estadísticos de las cronologías.

Statistical parameters of the chronologies.

\begin{tabular}{lccc}
\hline \multicolumn{1}{c}{ Especie } & Intercorrelación & Longitud de cronología & Sensibilidad \\
\hline Pinus durangensis & $0,60(P<0,01)$ & $1896-2015(120$ años $)$ & 0,34 \\
Pinus cooperii & $0,65(P<0,01)$ & $1890-2015(126$ años $)$ & 0,37 \\
Pinus lumholtzii & $0,60(P<0,01)$ & $1722-2015(294$ años $)$ & 0,23 \\
\hline
\end{tabular}


total, madera temprana y tardía (figura 4). Los meses de enero, marzo y abril mostraron una correlación significativa $(P<0,05)$ con la cronología de anillo total y madera temprana, siendo el mes de marzo el de mayor correlación ( $\mathrm{r}=0,57, P<0,01)$. La lluvia acumulada mostró correlaciones significativas $(P<0,01)$ para las tres cronologías (anillo total, madera temprana y tardía) en diferentes períodos a lo largo del año (figura 4B). Sin embargo, el perío- do estacional de lluvia acumulado enero-julio presentó la mayor correlación $\mathrm{r}=0,73(P<0,01), \mathrm{r}=0,72(P<0,01)$ y r $=0,67(P<0,01)$, para anillo total, madera temprana y madera tardía, respectivamente (figura 4). La cronología regional de anillo total presentó el mayor potencial para reconstruir la variabilidad de la lluvia estacional enero-julio, mayor asociación entre la cronología y los datos observados de lluvia $(\mathrm{r}=0,73, P<0,01)$.

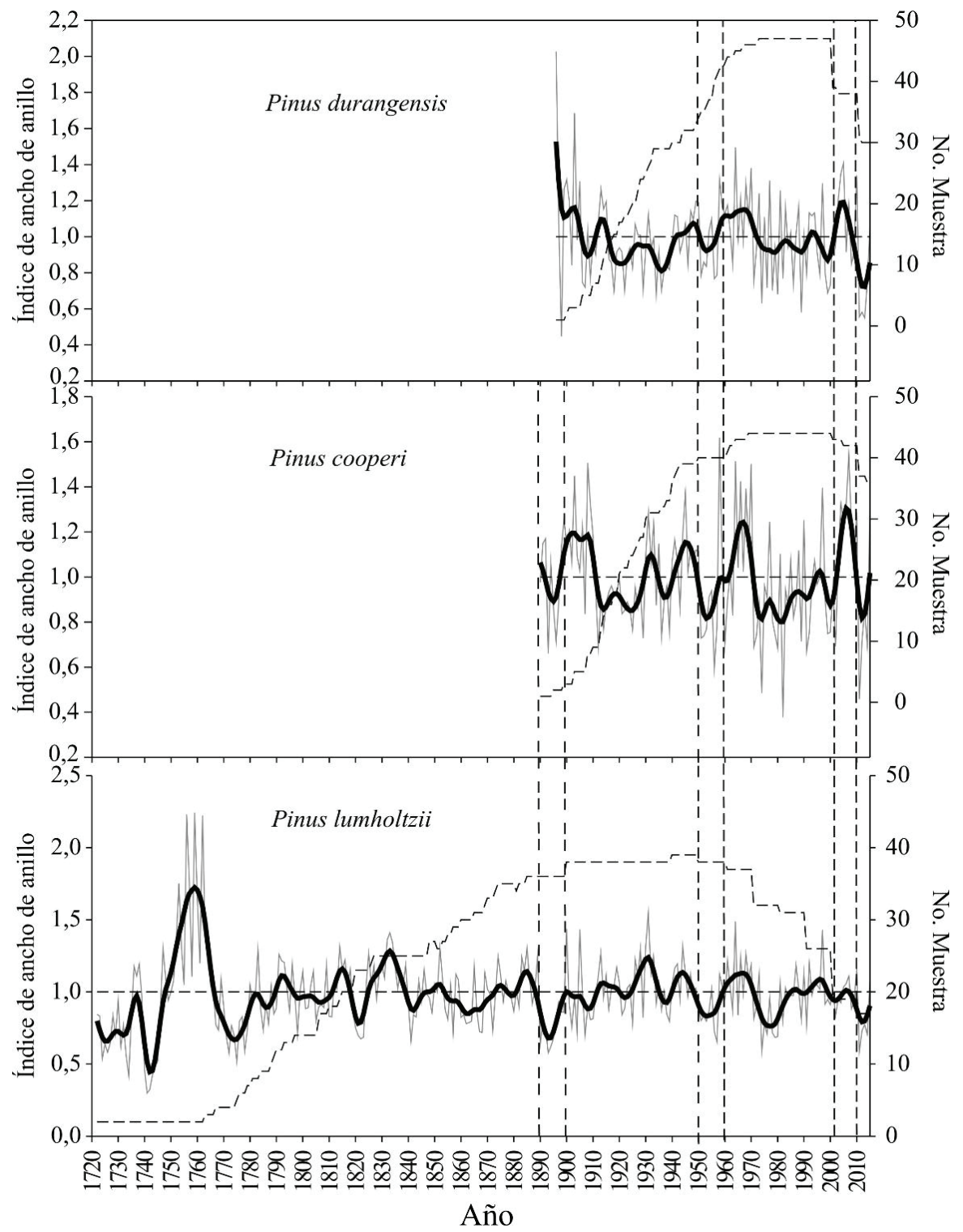

Figura 2. Cronologías generadas con base en el ancho de anillo de Pinus durangensis, Pinus cooperi y Pinus lumholtzii, las series muestran la variabilidad climática de baja y alta frecuencia de los últimos 300 años para la región de San Dimas, Durango. La línea en color gris de fondo representa la variabilidad anual, la línea horizontal punteada la media, la línea resaltada en color negro es un spline suavizado a 10 años para observar eventos de baja frecuencia y la línea punteada por arriba y debajo de la serie representa el tamaño de muestra para cada segmento de las cronologías. Las líneas verticales punteadas muestran la similitud en la variabilidad de las cronologías.

Chronologies generated based on the ring-width of Pinus durangensis, Pinus cooperi and Pinus lumholtzii; the series show climatic variability, low and high frequency of the last 300 years for San Dimas, Durango Region. The gray line in the background represents the annual variability, the horizontal dotted line is the mean, the highlighted black line is a spline smoothing to 10 years to observe low frequency events and the dotted lines above and below the series represent the sample size for each segment of the chronologies. The dotted vertical lines show the similarity in the variability of the chronologies. 

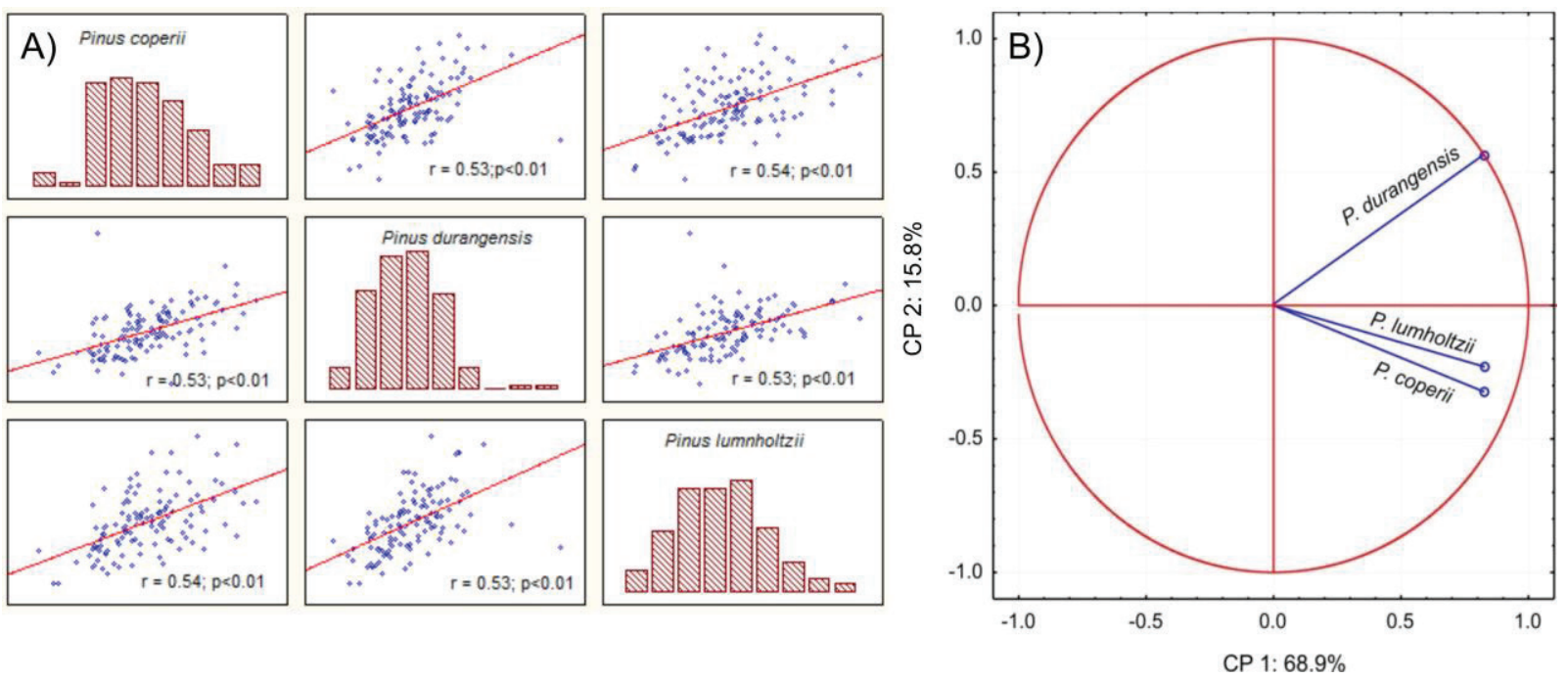

Figura 3. Matriz de correlación entre las tres cronologías (A) y análisis de componentes principales (PCA) que indicó la agrupación de las cronologías en el PC1, una misma variabilidad (B). Coeficientes del análisis de componentes principales (CP): i) cronología de Pinus cooperi: $\mathrm{CP} 1=0,84 ; \mathrm{CP} 2=-0,33$; ii) cronología de Pinus durangensis: $\mathrm{CP} 1=0,83$; CP2 = 0,56; iii) cronología de Pinus lumholtzii: $\mathrm{CP} 1=0,83 ; \mathrm{CP} 2=-0,23$.

Correlation matrix between the three chronologies (A) and principal component analysis (PCA) that indicated the grouping of chronologies in PC1, a same variability (B). Coefficients of the analysis of principal components (CP): i) chronology of Pinus cooperi: $\mathrm{CP} 1=0.84 ; \mathrm{CP} 2=-0.33$; (ii) chronology of Pinus durangensis: $\mathrm{CP} 1=0.83$; CP2 = 0.56; iii) chronology of Pinus lumholtzii: $\mathrm{CP} 1=0.83 ; \mathrm{CP} 2=-0.23$.
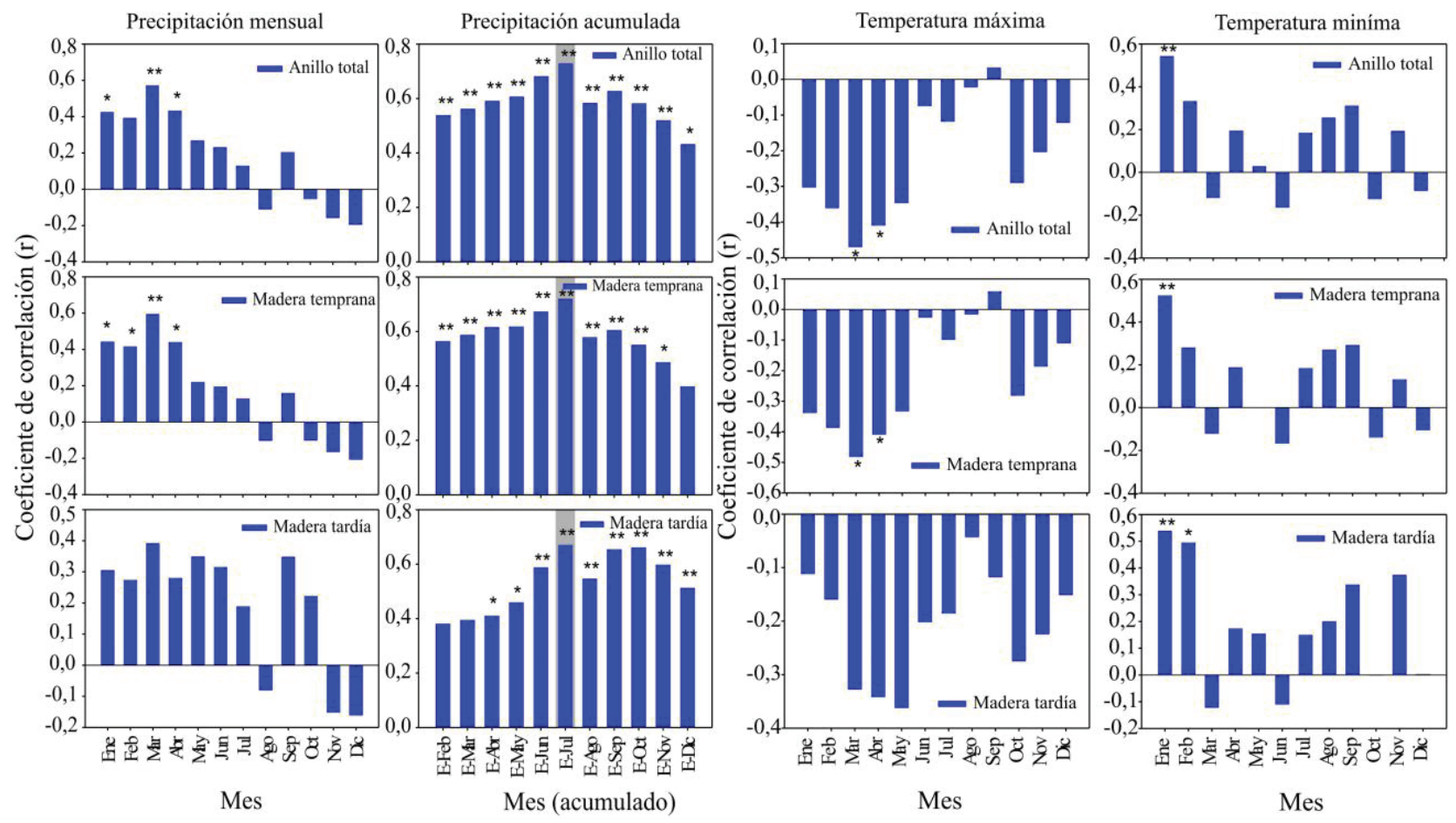

Figura 4. Análisis de función de respuesta entre variables climáticas (precipitación mensual, precipitación acumulada, temperatura máxima y temperatura mínima) y la cronología regional de anillo total, madera temprana y tardía. Donde $*=P<0,05 \mathrm{y} * *=P<0,01$ indican el grado de significancia de la correlación entre ambas variables.

Response function analysis between climatic variables (monthly rainfall, accumulated rainfall, maximum temperature and minimum temperature) and the ring-width regional chronology, earlywood and latewood. Where $* P<0.05$ and $* * P<0.01$ indicates the degree of significance of the correlation between the two variables. 
Con respecto a las variables temperatura máxima y mínima, los índices de crecimiento indicaron correlaciones negativas con la temperatura máxima (figura 4). Los meses de marzo y abril presentaron las correlaciones más bajas tanto para anillo total como para madera temprana, $\mathrm{r}=$ $-0,47,(P<0,05), \mathrm{r}=-0,41(P<0,05), \mathrm{r}=-0,48(P<0,05)$ y $\mathrm{r}=-0,41(P<0,05)$, respectivamente. Únicamente la temperatura máxima del mes de septiembre presentó una relación positiva pero no significativa $(P>0,05)$ con el crecimiento de las especies (figura 4). Caso contrario, la asociación entre los índices de las cronologías y la temperatura mínima mostraron correlaciones positivas para la mayoría de los meses del año (figura 4). La temperatura invernal de los meses enero-febrero indicó la correlación más alta, siendo el mes de enero la temperatura más importante en el crecimiento con valores de correlación de $\mathrm{r}=0,54(P<0,01), \mathrm{r}=0,52(P<0,01)$ y $\mathrm{r}=0,54(P<$ $0,01)$ para anillo total, madera temprana y tardía, respectivamente (figura 4). De igual manera, los meses de julioseptiembre y noviembre presentaron una influencia positiva no significativa $(P>0,05)$ en el crecimiento anual de Pinus durangensis, Pinus cooperi y Pinus lumholtzii (figura 4).

Las variables climáticas precipitación y temperatura influyeron de manera significativa $(P<0,05)$ a lo largo del año en el crecimiento de Pinus durangensis, Pinus cooperi y Pinus lumholtzii de la región de San Dimas, Durango. Sin embargo, la variable que resultó más importante en el crecimiento de las especies fue la precipitación. El índice regional de ancho de anillo presentó potencial para reconstruir la variabilidad de la lluvia estacional enero-julio, que explica el $52 \%(\mathrm{r}=0,73, P<0,01)$ de la variabilidad.

Reconstrucción de la precipitación. El periodo de datos observados (1991-2014) de precipitación estacional enero- julio presentó una asociación significativa con el índice regional de ancho de anillo $(\mathrm{r}=0,73, P<0,001)$, indicó potencial para generar un modelo de regresión y reconstruir la variabilidad de la precipitación invierno-primavera (figura 5A). El modelo de regresión lineal generado para la reconstrucción [1] se consideró estadísticamente $(P<0,001)$ aceptable como se observa en los cuadros 2 y 3 .

$$
Y_{t}=75,4751+391,0180^{*} X_{t}
$$

Donde:

$\mathrm{Y}_{t}=$ Valor de precipitación $(\mathrm{mm})$ enero-julio reconstruido para un año $t$.

$\mathrm{X}_{t}=$ Índice de ancho anillo para un año $t$.

Calibración y verificación del modelo. La calibración (subperiodo 2005-2014) mostró una correlación altamente significativa $\mathrm{r}=0,85, P<0,001$, la cual explicó el $72 \%$ de la variabilidad de la lluvia (figura $5 \mathrm{C}$ ). La verificación (subperiodo 1991-2004) indicó una correlación altamente significativa $\mathrm{r}=0,64, P<0,001$, que explicó el $41 \%$ de la variabilidad de la lluvia.

Dado que ambos subperiodos, tanto la calibración como la verificación del modelo mostraron una relación significativa, se empleó el lapso total de datos climáticos disponibles (1991-2014) para generar la ecuación de reconstrucción, que tuvo una $\mathrm{r}=0,73\left(\mathrm{r}^{2}=0,53, P<0,01\right)$ (cuadro 3, figura 5C).

Variabilidad de la precipitación reconstruida. Se reconstruyó la precipitación invierno-primavera para los pasados 294 años, que comprendió el período de 1722-2015. La serie indicó una alta variabilidad interanual y multianual, que históricamente ha caracterizado la precipitación estacional inviernoprimavera para la región de San Dimas, Durango (figura 6).

Cuadro 2. Modelo generado para la reconstrucción de la precipitación enero-julio.

Regression model for the reconstruction of January-July precipitation.

\begin{tabular}{|c|c|c|c|c|}
\hline Período & Coeficientes & Error estándar & Prueba de $t$ & $P$ \\
\hline \multirow[t]{2}{*}{$1991-2014$} & a 75,4751 & 78,26444 & 0,964360 & 0,000001 \\
\hline & b $\quad 391,0180$ & 78,24125 & 4,997594 & 0,000001 \\
\hline
\end{tabular}

Cuadro 3. Análisis de varianza del modelo generado.

Analysis of variance for the regression model generated.

\begin{tabular}{cccccc}
\hline Fuente de variación & Suma de cuadrados & g.l. & Cuadrados medios & F & $P$ \\
\hline Regresión & $159.314,2$ & 1 & $159.314,2$ & 24,97 & 0,000001 \\
Residual & $140.331,6$ & 22 & $6.378,7$ & - & - \\
\hline Total & $299.645,8$ & - & - & - & - \\
\hline
\end{tabular}


En los últimos tres siglos se reconstruyeron importantes eventos de fuerte sequía para los períodos de 1740-1746, 1766-1780, 1785-1786, 1819-1824, 1890-1900, 19351940, 1950-1957 y 2011-2015 (figura 6). Por su extensión y extrema sequía sobresalieron los períodos 1766-1780 (15 años), 1890-1900 (11 años) y 1950-1957 (8 años).
Influencia ENSO sobre el clima. El análisis espectral de coherencia de ondeletas al asociar la serie de precipitación reconstruida invierno-primavera (figura 7A) y el índice del NIÑO 3 SST (figura 7B), permitió observar, que el espectro de ondeleta para la precipitación reconstruida invierno-primavera, mostró áreas significativas $(P<0,05)$ que
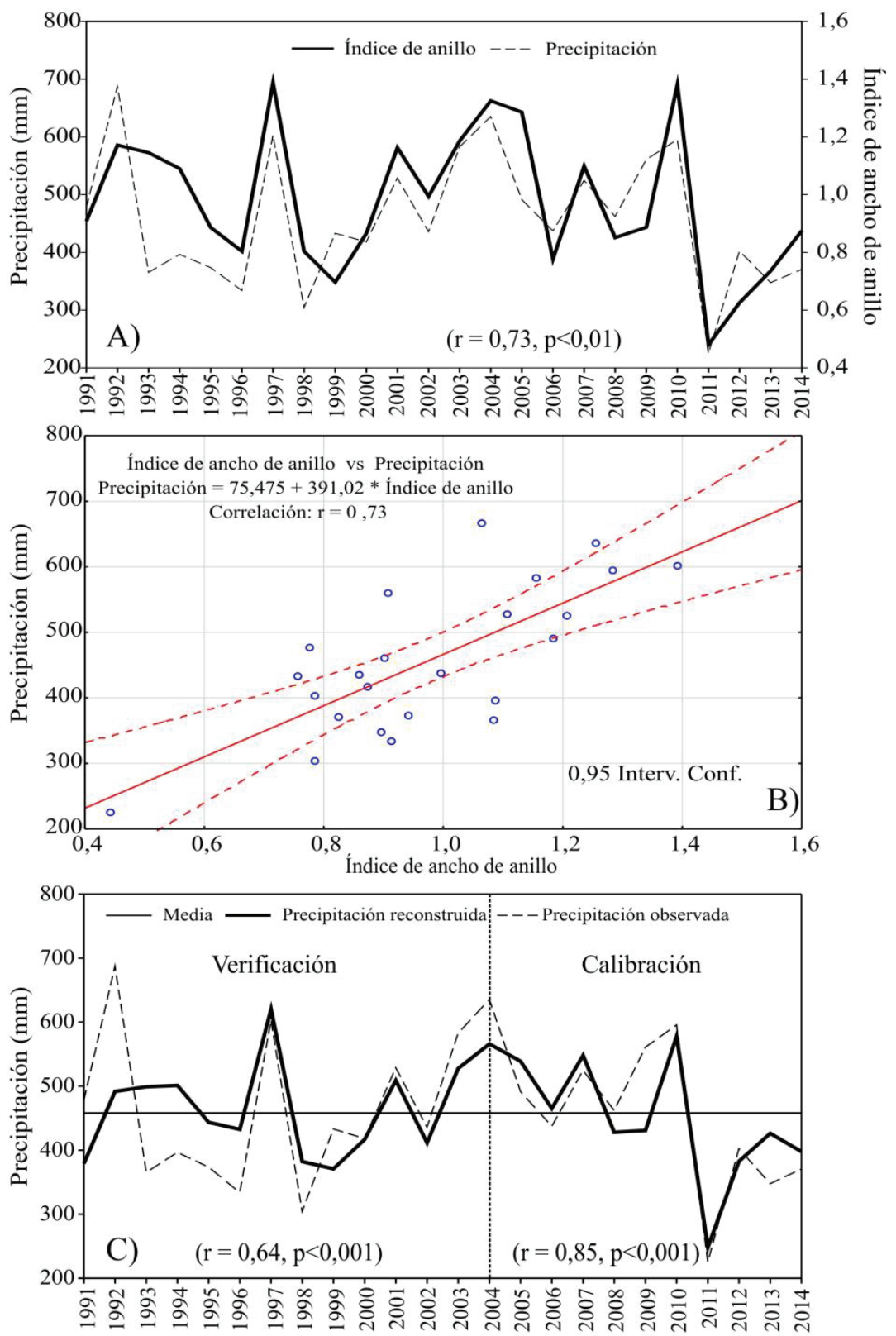

Año

Figura 5. Asociación entre la precipitación estacional enero-julio y el índice regional de ancho de anillo que comprendió el período de 1991-2014 (A), modelo de regresión lineal entre las dos variables desarrollado empleando el programa STATISTICA Kernel Release 5.5 (Stat Soft Inc. 2000) (B) y comparación de la precipitación estacional reconstruida enero-julio (línea sólida) y la precipitación observada (línea punteada) para el período 1991-2014 (24 años), verificación y calibración del modelo de regresión (C).

Association between seasonal rainfall January-July and the regional ring width index covering the period of 1991-2014 (A), linear regression model between the two variables generated using the program STATISTICA Kernel release 5.5 (Stat Soft Inc. 2000) (B) and comparing the reconstructed seasonal precipitation January-July (solid line) and observed precipitation (dotted line) for the period 1991-2014 (24 years), and verification and calibration of the regression model (C). 


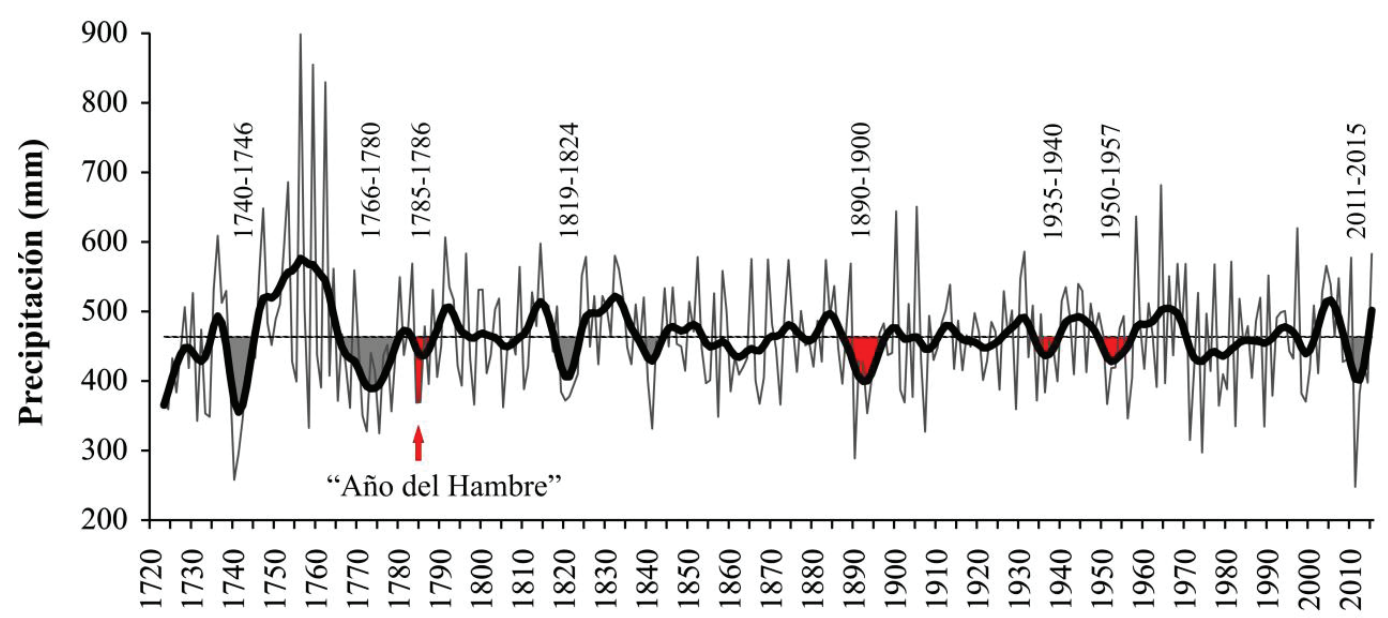

Año

Figura 6. Reconstrucción de la variabilidad de la precipitación estacional invierno-primavera de los últimos tres siglos (294 años) para la región de San Dimas, Durango. La línea gris de fondo indica la variabilidad anual, la línea resaltada en color negro representa una curva decenal flexible que permite observar eventos de baja frecuencia (sequías y períodos húmedos) y la línea horizontal representa la lluvia promedio. Relleno en color gris se resaltan las sequías más fuertes en los últimos 300 años para esta región del norte de México, y en color rojo cuatro de las sequías más severas documentadas por archivos históricos para el centro del país.

Reconstruction of the variability of seasonal precipitation winter-spring of the last three centuries (294 years) for the region of San Dimas, Durango. The bottom gray line indicates the annual variability, the line highlighted in black represents a flexible decennial curve that permitted to observe low frequency events (droughts and wet periods) and the horizontal line represents the average rainfall. The grey-filled area represents the strongest droughts in the past 300 years for this region of northern Mexico, and in red four of the most severe droughts documented by historical archives to the center of the country.

corresponden a periodos anuales, bianuales, tetra-anuales, de 5, 11 y 16 años. Mientras que el espectro de ondeleta de ENSO mostró su ciclicidad irregular de 1 a 7 años y de 10 a 12 años.

Se determinó una coherencia significativa $(P<0,05)$ de la variabilidad climática regional y el índice del NIÑO 3 SST durante el periodo $1770-1978$ a frecuencias de 1 a 5 años, para todo el período ambos fenómenos coinciden en fase (figura 7C). Así mismo, se observaron períodos en fase para $1800-1830,1810-1860$ y $1770-1910$, con frecuencias de 10-14, 20-27 y 40-52 años, respectivamente. Los periodos de sequía más extensos reconstruidos para la región de San Dimas, Durango, en los últimos 294 años (1766-1780, 1819-1824, 1890-1900 y 1950-1957) fueron modulados de manera significativa $(P<0,05)$ por la ocurrencia de ENSO (figura 7C).

\section{DISCUSIÓN}

Sensibilidad de las series. Las tres cronologías desarrolladas en la región de San Dimas, Durango, presentan valores de correlación entre series que oscilan de $0,60-0,65$ $(P<0,01)$ y una sensibilidad media de $0,23-0,37$ (cuadro 1), el programa COFECHA (Holmes 1983) establece como mínima correlación significativa entre muestras de $\mathrm{r}$ $=0,3281(P<0,01)$. Los valores estadísticos de las series son similares y en algunos casos superiores a otras especies de la Sierra Madre Oriental, como Pinus cembroides
Zucc. (Constante et al. 2009), Pinus pinceana Gordon (Santillán et al. 2010) y Pinus hartwegii Lindl. (Villanueva et al. 2015); y de la Sierra Madre Occidental Pinus cooperi Blanco (Cerano et al. 2012a), que se han empleado con éxito en la reconstrucción de variables climáticas. Lo anterior confirma el potencial de las series de Pinus durangensis, Pinus cooperi y Pinus lumholtzii (figura 2) para ser consideradas proxy de la variabilidad climática de la región y desarrollar estudios paleoclimáticos.

Variabilidad común de las series. Las tres cronologías presentan una variabilidad climática común a través del tiempo, lo que sugiere que el crecimiento anual de las tres especies está influenciado por condiciones climáticas que modulan el clima regional. Estudios climáticos para el norte de México, reportan que el clima en esta región es modulado de manera significativa $(P<0,01)$ por El Niño Oscilación del Sur (ENSO, por sus siglas en inglés) (Stahle et al. 1998, Magaña et al. 1999, Cerano et al. 2012a), a lo que se atribuye la misma variabilidad entre series de diferentes especies. Resultados similares sobre una respuesta común entre cronologías de diferentes especies se han reportado para la Sierra Madre Oriental, atribuible a una variabilidad climática de gran escala (Cerano et al. 2011). Así mismo, para la Sierra Madre Occidental, se ha determinado una variabilidad común entre cronologías de la misma especie separadas por kilómetros de distancia, pero condiciones climáticas comunes (Cerano et al. 2012b, Villanueva et al. 2015). 
Respuesta de la cronología a las variables climáticas. La reconstrucción de la precipitación para la región de San Dimas, Durango, se fundamentó en el crecimiento anual como variable independiente, cuya formación está relacionada significativamente $(\mathrm{r}=0,73, P<0,01)$ con la preci- pitación invierno-primavera. Una explicación a esta respuesta es, que los inviernos más húmedos contribuyen a un mejor proceso de fotosíntesis en la estación cálida y, por tanto, en mayor crecimiento de los árboles (Kerhoulas et al. 2013). Las lluvias en invierno suelen ser de baja inten-

A) Precipitación reconstruida invierno-primavera

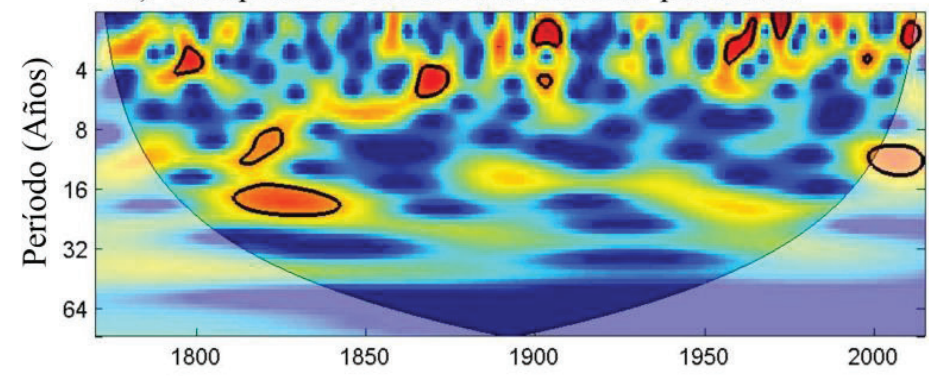

B) Índice NIÑO 3 SST
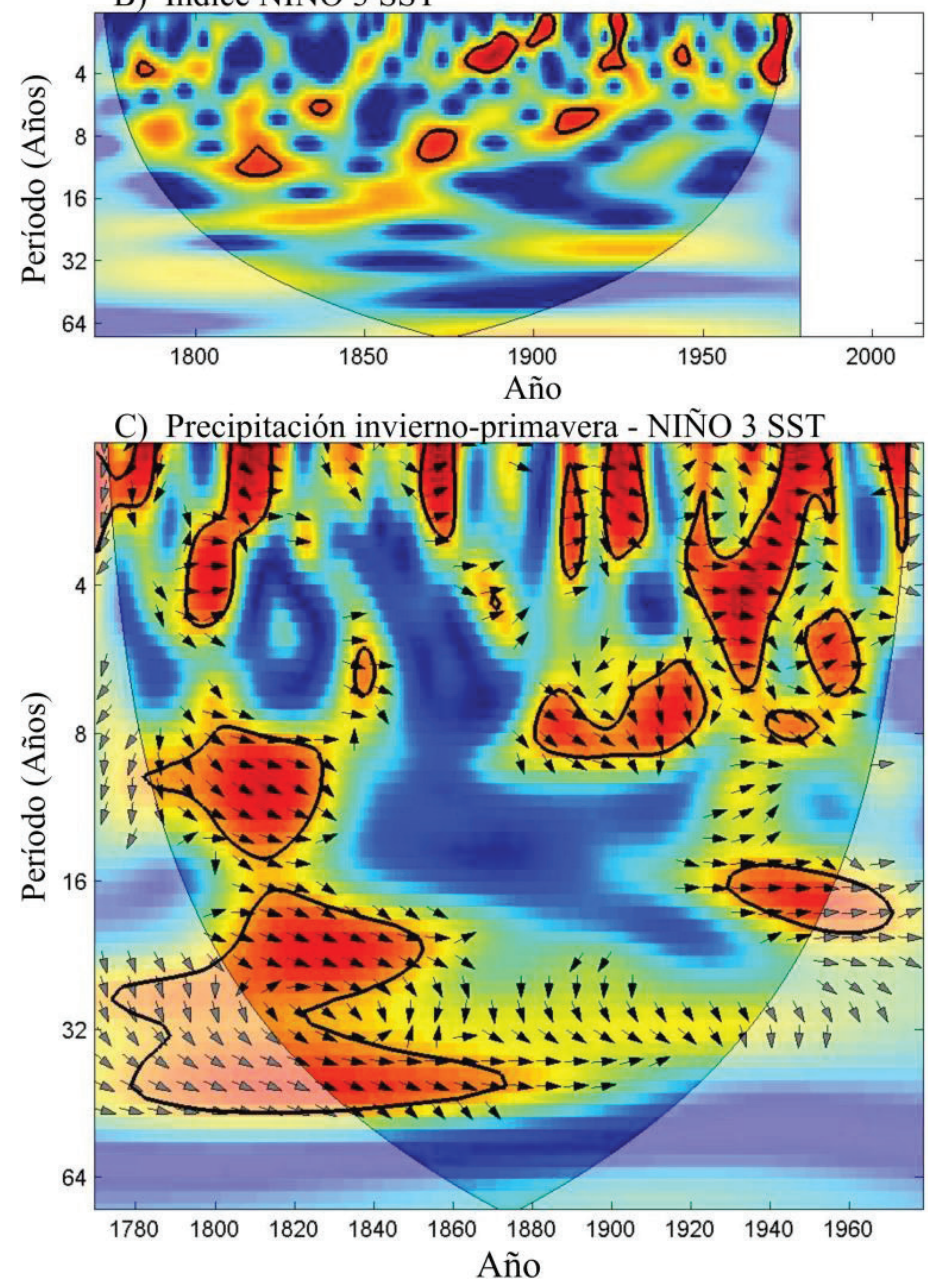

Figura 7. Espectros potenciales de ondeleta a nivel anual, precipitación reconstruida invierno-primavera A), índice NIÑO 3 SST B) y C) representa el dominio de coherencia de ondeleta entre la serie de precipitación reconstruida y el índice NIÑO 3 SST. Las manchas en color rojo delimitadas por una línea de color negro, representan períodos con frecuencias significativas $(P<0,05)$ y las flechas orientadas hacia la derecha indican que ambos fenómenos están en fase (correlación positiva).

Potential wavelet spectrum at annual level, winter-spring precipitation reconstructed A), NIÑO 3 SST index B) and C) represents the wavelet coherence domain between the reconstructed precipitation series and NIÑO 3 SST index. Red spots bounded by a black line represent periods with significant frequency $(P<0.05)$. Right oriented arrows indicate that both phenomena are in phase (positive correlation). 
sidad, lo que favorece la infiltración, saturación del suelo y una baja evapotranspiración, resultando en un balance hídrico positivo que es empleado por el árbol al inicio de la estación de crecimiento (Fritts 2001).

La falta de una asociación significativa $(P>0,05)$ entre el crecimiento anual y la precipitación de verano, se puede atribuir a que antes del período de verano, el anillo anual presenta una formación alrededor del $60 \%$; además, en este periodo del año las lluvias superan la capacidad de infiltración del suelo y el agua se escurre como flujo superficial, no es registrada en el crecimiento del árbol (García 1978).

La respuesta del crecimiento de diferentes coníferas a la precipitación invernal en la región norte de México ha sido documentada y discutida ampliamente en diversos estudios paleoclimáticos (Cleaveland et al. 2003, Villanueva et al. 2007, Cerano et al. 2009, 2011, 2012a).

Con respecto a las variables de temperatura, existe una relación negativa significativa $(P<0,05)$, entre las temperaturas máximas y el crecimiento del anillo total y el de la madera temprana en los meses de marzo y abril (figura 4). Esto se explica por el hecho de que en estos meses ocurren los valores más extremos de las temperaturas máximas (33 ${ }^{\circ} \mathrm{C}$ ) que se asocia a la baja humedad relativa que ocurre y que caracterizan la temporada de mayor riesgo de incendios forestales. Temperaturas elevadas del aire por encima de $25^{\circ} \mathrm{C}$ disminuyen drásticamente la apertura de estomas, en consecuencia, también se reduce la asimilación de fotosintatos (Leverenz 1981). Otra explicación, es que temperaturas elevadas incrementan el déficit de vapor de agua en la capa fronteriza de las acículas, en el orden de 2,0 a $3,0 \mathrm{kPa}$, lo que induce una reducción en la apertura de los estomas, alcanzando su cierre total a los $3,5 \mathrm{kPa}$ cuando la temperatura del aire sobrepasa los $30{ }^{\circ} \mathrm{C}$, afectando la transpiración y la difusión de $\mathrm{CO}_{2}$ (Leverenz 1981).

Existe una relación positiva, altamente significativa $(P<0,01)$, entre las temperaturas mínimas y el crecimiento del anillo total, madera temprana y tardía en el mes de enero, dicha relación solo es significativa $(P<0,05)$ para la madera tardía en el mes de febrero (figura 4). Lo anterior se puede atribuir a que en estos meses se registran las temperaturas mínimas promedio más bajas, $-9,2$ y - 8,6 , respectivamente. Lo cual se relaciona con una menor evaporación y por lo tanto mayor humedad en el suelo, agua que el árbol tiene disponible para emplear al inicio de la estación de crecimiento.

Sequías reconstruidas. La disponibilidad de información histórica que permita analizar eventos climáticos extremos, su recurrencia y los estragos propiciados a la sociedad son escasos, y para algunas regiones del norte de México nulos. Las series paleoclimáticas son el proxy que permite analizar varios siglos en el tiempo la variación del clima y sus efectos a diferentes escalas geográficas.

Documentos históricos reportan para el centro de México, siete episodios $(1550,1594-1604,1692-1713,1785-$ 1786, década de 1830, 1891-1902 y la década de 1950) bien documentados de sequía, hambre y agitación social (Florescano 1980, 1986). Siete períodos graves y prolongados que se asocian a un pobre rendimiento de maíz después del año 1500 (Therrell et al. 2006).

Para la región de San Dimas, Durango, la reconstrucción de la variabilidad de la lluvia de los pasados 294 años (1722-2015), registra los últimos cuatro eventos más severos de sequía (1785-1786, la década de 1830, 18911902 y la década de 1950) documentados para el centro de México (Florescano 1980, 1986). Estas sequías no son eventos localizados de una región, las series paleoclimáticas permiten observar la amplitud geográfica de eventos climáticos extremos, la severidad de sus efectos negativos y comparar entre regiones.

El período de 1785-1786 representa la primera de cuatro sequías ampliamente documentadas, 1785 dio lugar a una terrible hambruna que se agudizo en 1786, comúnmente conocida como "El Año del Hambre" (Florescano 1986, figura 6). Este fenómeno se describe como el acontecimiento más desastroso en toda la historia de la agricultura del maíz en la época colonial, esta hambruna al parecer también condujo a un brote de enfermedad y epidemia que mató a unas 300.000 personas (Florescano 1986). Esta extraordinaria carencia de alimentos, combinada con la epidemia tuvo un efecto devastador en la economía y la sociedad de la Nueva España. Florescano (1986) ha planteado la hipótesis de que este episodio contribuyó a los disturbios civiles que pudieron haber influido en el movimiento de independencia de 1810. Aunque este evento se extendió hasta la región norte del país, reconstrucciones para el centro de México (Therrell et al. 2006), muestran un efecto más severo comparado con esta parte del estado de Durango. Por el contario, la sequía reconstruida pocos años atrás (1766-1780) parece haber tenido un mayor impacto para esta región de Durango que para el centro del país.

Debido a la agitación y el desorden que siguió a la Guerra de la Independencia que duró desde 1810 hasta 1821, los registros históricos son menos accesibles (O'Hara y Metcalfe 1995). Sin embargo, al igual que para el centro del país, se corrobora la condición de sequía que prevaleció de 1817-1823 para esta región de Durango, y se reafirma la amplitud de los estragos negativos (sequías). Florescano (1980) se refiere a una grave sequía y la escasez de cultivos en el Valle de México durante la década de 1830, especialmente en la primavera de 1836, y los registros eclesiásticos indican una sequía en el período 1838-1841. La reconstrucción para el norte de México, confirma estas observaciones, indica una década de déficit en las lluvias 1835-1845 (figura 6). Así mismo, se corrobora que para años específicos el clima es modulado a gran escala por fenómenos climáticos comunes.

Una disminución de la lluvia se reconstruyó de nuevo para 1890-1900 (figura 6). De acuerdo con referencias históricas ocurrió una extraordinaria sequía y cosechas deficientes en gran parte de México a partir de 1891 y hasta 1906 (Florescano 1980). Un periodo considerado como 
sequía nacional, es el intervalo de tiempo de 1890-1895, seis años en los cuales la agricultura, ganadería y personas se vieron fuertemente afectados en la mayor parte de México (Florescano 1980). Este periodo de sequías severas de finales del siglo XIX es reportado en otras reconstrucciones para el norte de México, en los estados de Coahuila, Durango, Chihuahua y Baja California Sur (Pohl et al. 2003, Cleaveland et al. 2003, Cerano et al. 2011, 2012a). O'Hara y Metcalfe (1995) sugieren que las condiciones de déficit de precipitaciones y la escasez de alimentos durante los primeros 15 años del siglo XX también pueden haber contribuido al malestar social que resultó en la Revolución Mexicana de 1910.

Finalmente, las intensas sequías de la década de 1950 (1950-1957), son uno de los eventos secos que han impactado severamente la región norte de México. La década de 1950, se considera una sequía extrema que impactó a México y gran parte del suroeste de los Estados Unidos (Florescano y Swam 1995). Los años de 1950, 1951, 1953, 1956 y 1958, se reportan como años con sequía severa y 1957 como sequía extremadamente severa. Dichas sequías han impactado no únicamente a México, las sequías de los años 1950, 1956 y 1957 impactaron en toda América (Florescano 1980).

Las sequías de 1956 y 1957, ocasionaron desempleo y migración, así como movilizaciones campesinas. La sequía de 1956, afectó esencialmente el norte de México, afectando a 60.000 campesinos de los cuales muchos tuvieron que emigrar a los Estados Unidos. Esta crisis económica y ocupacional fue particularmente grave en la Comarca Lagunera, la carencia de agua paralizó las labores agrícolas y se incrementó de igual manera la emigración laboral (Florescano 1980).

Influencia ENSO sobre el clima. Los eventos de sequía más fuertes reconstruidos para esta región del estado de Durango, son episodios registrados en gran parte del país, con variaciones en intensidad entre regiones, lo cual obedece a que el clima es modulado a gran escala por fenómenos climáticos comunes. Así mismo, se ha determinado una frecuencia o recurrencia de eventos extremos de sequía aproximadamente cada 50 años, cada mediado y final de siglo.

Los eventos de baja frecuencia con intervalos de 50 años aproximadamente, son modulados de manera significativa $(P<0,05)$ por la variabilidad del ENSO. Estos resultados coinciden con lo reportado tanto para el norte (Cerano et al. 2011) como para el centro (Therrell et al. 2006) de México, eventos extremos de sequías presentan una recurrencia de 50 años. Con base en estos resultados se evidencia una relación en fase significativa $(P<0,05)$ del ENSO y la variabilidad de la lluvia. En los últimos tres siglos el ENSO ha influido de manera significativa en la variabilidad de lluvia para esta región de San Dimas, Durango. El fenómeno ENSO actualmente se puede predecir con antelación, por lo tanto, el conocimiento de cómo este fenómeno modula la variabilidad climática, representa in- formación esencial para los manejadores de los recursos naturales y tomadores de decisiones, a quienes les permitirá saber con anticipación su efecto negativo (Niña-Sequía) o positivo (Niño-Lluvias) y poder tomar las acciones necesarias para prevenir y reducir condiciones adversas en las actividades productivas y la sociedad.

\section{CONCLUSIONES}

Las especies Pinus cooperi, Pinus durangensis y Pinus lumholtzii presentan potencial para desarrollar series paleoclimáticas, sus anillos de crecimiento anual representan un excelente proxy del clima, que permitirán generar información en regiones donde se carece de registros observados. Se acepta la primera hipótesis de investigación, que establece que los crecimientos anuales de las tres especies presentan potencial para reconstruir la variabilidad climática que ha caracterizado en los últimos siglos a la región de San Dimas, Durango.

Se observa una influencia significativa del ENSO en la variabilidad de la precipitación para esta región del estado de Durango, resultados que sustentan la segunda hipótesis de investigación, que plantea que el ENSO modula la variabilidad climática histórica en esta región del norte del país. En los últimos tres siglos, los eventos extremos de sequía muestran patrones recurrentes cada 50 años aproximadamente, cada mediados y finales de siglo, asociados de manera significativa a la variabilidad del ENSO.

Es importante dar continuidad al desarrollo de series paleoclimáticas con base en Pinus cooperi, Pinus durangensis y Pinus lumholtzii, desarrollar una red de cronologías que permita extender los registros climáticos y con una base de datos más robusta analizar a mayor escala la extensión, severidad y recurrencia de eventos climáticos extremos.

\section{AGRADECIMIENTOS}

Esta investigación fue posible gracias al financiamiento CONAFOR-CONACYT a través del proyecto "Variabilidad climática e interacción con otros factores que afectan la dinámica poblacional de descortezadores en bosques amenazados de México", con registro: CONAFOR-2014, C01-234547.

\section{REFERENCIAS}

Bradley RS. 1999. Paleoclimatology. Reconstruction climates of the Quaternary. Amsterdam. Harcourt Academic Press. 613 p.

Cerano PJ, DJ Villanueva, PZ Fulé, AJG Arreola, CI Sánchez, CRD Valdez. 2009. Reconstrucción de 350 años de precipitación para el suroeste de Chihuahua, México. Madera y Bosques 15(2): 27-44.

Cerano PJ, DJ Villanueva, CRD Valdez, GJ Méndez, GV Constante. 2011. Sequías reconstruidas en los últimos 600 años para el noreste de México. Revista Mexicana de Ciencias Agrícolas 2: 235-249. 
Cerano PJ, DJ Villanueva, CRD Valdez, GV Constante, BJL González, AJ Estrada. 2012a. Precipitación reconstruida para la parte alta de la cuenca del Río Nazas, Durango. Revista Mexicana de Ciencias Forestales 3(10): 7-23.

Cerano PJ, GM Rivera, AJ Estrada, CR Trucíos, SJC Ríos. 2012b. Análisis dendrocronológico de Pinus cooperi en Durango, México. Agrofaz 12(3): 81-88.

Cleaveland MK, DW Stahle, MD Therrell, DJ Villanueva, BT Burns. 2003. Tree-ring reconstructed winter precipitation in Durango, Mexico. Climatic Change 59(3): 369-388.

Constante GV, DJ Villanueva, PJ Cerano, OEH Cornejo, M Valencia. 2009. Dendrocronología de Pinus cembroides Zucc. y reconstrucción de precipitación estacional para el Sureste de Coahuila. Ciencias Forestales en México 34(106): 17-39.

Florescano ME. 1980. Análisis histórico de las sequías en México. México. Secretaría de Agricultura y Recursos Hidráulicos. $158 \mathrm{p}$.

Florescano ME. 1986. Precios del maíz y crisis agrícolas en México: 1708-1810. Distrito Federal, México. Ediciones Era. $236 \mathrm{p}$.

Florescano ME, S Swam. 1995. Breve historia de la sequía en México. Xalapa, México. Universidad Veracruzana. 246 p.

Fritts HC. 2001. Tree rings and climate. New Jersey, USA. Blackburn Press Caldwell. 567 p.

García E. 1978. Apuntes de climatología. México DF, México. UNAM. 153 p.

Holmes RL. 1983. Computer-assisted quality control in tree-ring dating and measurement. Tree-Ring Bulletin 43: 69-78.

INEGI (Instituto Nacional de Estadística y Geografía, MX). 2010. Prontuario de información geográfica municipal de los Estados Unidos Mexicanos, San Dimas, Durango, Clave geostadistica10026. Disponible en: http://www3.inegi.org. $\mathrm{mx} /$ sistemas/méxicocifras/datos-geograficos/10/10026.pdf

Kerhoulas LP, TE Kolb, GW Koch. 2013. Tree size, stand density, and the source of water used across seasons by ponderosa pine in northern Arizona. Forest Ecology Management 289: 425-433.

Leverenz JW. 1981. Photosynthesis and transpiration in large forest-grown Douglas-fir: diurnal variation. Canadian Journal of Botany 59(3): 349-356.

Magaña V, JL Pérez, JL Vázquez, E Carrizosa, J Pérez. 1999. El Niño y el clima. In Magaña V ed. Los impactos de El Niño en México. México. SEP-CONACYT. 23-68.

Mathworks. 2002. Documentation-Signal Processing Toolbox. Versión 6.5. MATLAB.
O'Hara SL and SE Metcalfe. 1995. Reconstructing the climate of Mexico from historical records. The Holocene 5(4): 485-490.

Perry Jr JP, A Graham, MD Richardson. 1998. The history of pines in México and Central America. In Richardson DM ed. Ecology and Biogeography of Pinus. Cambridge, UK. Cambridge University Press. p. 137-147.

Pohl K, MD Therrell, JS Blay, N Ayotte, JJ Cabrera, SC Díaz, EH Cornejo, JA Elvir, ME González, D Opland, J Park, G Pederson, SS Bernal, LS Vázquez, DJ Villanueva, DW Stahle. 2003. A cool season precipitation reconstruction for Saltillo, Mexico. Tree-ring Research 59(1): 11-19.

Santillán HM, OE Cornejo, DJ Villanueva, PJ Cerano, MS Valencia, AMA Capó. 2010. Potencial dendroclimático de Pinus pinceana Gordon en la Sierra Madre Oriental. Madera y Bosques 16(1): 17-30.

Stahle DW, RD D'Arrigo, PJ Krusic, MK Cleaveland, ER Cook, RJ Allan, JE Cole, RB Dunbar, MD Therrell, DA Gay, MD Moore, MA Stokes, BT Burns, J Villanueva-Díaz, LG Thompson. 1998. Experimental dendroclimatic reconstruction of the Southern Oscillation. Bulletin of the American Meteorological Society 79(10): 2137-2152.

Stahle DW, DJ Villanueva, DJ Burnette, PJ Cerano, RR Heim Jr, FK Fye, SR Acuña, MD Therrell, MK Cleaveland, DK Stahle. 2011. Major Mesoamerican droughts of the past mi1lennium. Geophysical Research Letters 38(L05703): 1-4.

Stat Soft Inc. 2000. Statistica Software, Kernel Release version 5.5 A. 1984-2000. Tulsa, Oklahoma, USA.

Stokes MA, TL Smiley. 1996. An Introduction to tree-ring dating. Tucson, USA. University of Arizona Press. 73 p.

Therrell MD, DW Stahle, DJ Villanueva, OE Cornejo, MK Cleaveland. 2006. Tree-ring reconstructed maize yield in central Mexico: 1474-2001. Climate Change 74: 493-504.

Villanueva DJ, DW Stahle, BH Luckman, PJ Cerano, MD Therrell, MK Cleveland, OE Cornejo. 2007. Winter-spring precipitation reconstruction from tree rings for northeast Mexico. Climate Change 83(1): 117-131.

Villanueva DJ, PJ Cerano, SL Vázquez, DW Stahle, PZ Fulé, LL Yocom, OF Ramos, CJA Ruiz. 2015. Red dendrocronológica del pino de altura (Pinus hartwegii Lindl.) para estudios dendroclimáticos en el noreste y centro de México. Investigaciones Geográficas, Boletín del Instituto de Geografía, UNAM 86: 5-14.

Woodhouse CA, JJ Lukas. 2006. Multi-century tree-ring reconstructions of Colorado streamflow for water resource planning. Climate Change 78(2-4): 293-315. 
